\title{
Raman analysis of phonon lifetimes in AIN and GaN of wurtzite structure
}

\author{
Leah Bergman, Dimitri Alexson, Patrick L. Murphy, and Robert J. Nemanich \\ Department of Physics, North Carolina State University, Raleigh, North Carolina 27695-8202 \\ Mitra Dutta and Michael A. Stroscio \\ U.S. Army Research Office, P.O. Box 12211, Research Triangle Park, North Carolina 27709-2211 \\ Cengiz Balkas, Hyumin Shin, and Robert F. Davis \\ Department of Materials and Science Engineering, North Carolina State University, Raleigh, North Carolina 27695
}

(Received 28 September 1998)

\begin{abstract}
Raman analyses of the lifetimes of phonons in GaN and AlN crystallites of wurtzite structure are presented. In order to ensure the accuracy of the measurement of the phonon lifetimes, an experimental procedure to eliminate the broadening due to the finite slit width was performed. The lifetime analyses indicate that the phonon lifetimes in AlN as well as in GaN fall into two main time regimes: a relatively long time of the $E_{2}^{1}$ mode and much shorter times of the $E_{2}^{2}, E 1(\mathrm{TO})$, and $A 1(\mathrm{TO})$ modes. The lifetimes of the $E_{2}^{1}, E_{2}^{2}, E 1(\mathrm{TO})$, $A 1(\mathrm{TO})$, and $A 1(\mathrm{LO})$ modes of an high-quality AlN crystallite are 4.4, 0.83, 0.91, 0.76, and $0.45 \mathrm{ps,} \mathrm{respec-}$ tively. Moreover, the lifetime of the $A 1$ (LO) mode found in this study is consistent with the current phonondecay model of that mode in wurtzite structure materials. The lifetimes of $E_{2}^{1}, E_{2}^{2}, E 1(\mathrm{TO})$, and $A 1(\mathrm{TO})$ of a $\mathrm{GaN}$ crystallite were found to be $10.1,1.4,0.95$, and $0.46 \mathrm{ps}$, respectively. The $A 1$ (LO) mode in the GaN was not observed and its absence is attributed to plasmon damping. The lifetime shortening due to impurities was also studied: the lifetimes of the Raman modes of an AlN crystallite, which contains about two orders of magnitude more $\mathrm{Si}$ and $\mathrm{C}$ impurities relative to the concentration of the high-quality crystallite were found to be $50 \%$ shorter. [S0163-1829(99)04419-7]
\end{abstract}

\section{INTRODUCTION}

The realization of device fabrication using AlN, GaN, and their alloy systems as the constituent materials, has led to numerous investigations concerning growth as well as analytical techniques to study and improve these wide-bandgap family semiconductors. ${ }^{1}$ Raman spectroscopy has proven useful in analyzing the properties of wide-bandgap materials: it reveals information about sample quality as well as phonon interactions and dynamics. ${ }^{2-10}$

One crucial aspect impacting device performance is phonon interaction with free carriers. In general, the interactions can degrade the viability of the device; however, recent studies have demonstrated that the phonon interactions may be utilized to engineer certain laser devices. ${ }^{11-12}$

The phonon lifetimes are important in both these effects, and one principal way of measuring these lifetimes is via Raman spectroscopy. It has been demonstrated previously that phonon lifetimes and the factors affecting them can be calculated from the Raman linewidth. Among the materials that have been analyzed via their Raman linewidth are diamond, Si, $c$-BN, GaAs, and InP. ${ }^{13-17}$

In general, the theory of a spectral line shape of a signal in a dispersive medium predicts the line shape to be Lorentzian and the linewidth, a parameter describing the damping effect, to be inversely proportional to the lifetime of the signal. ${ }^{18}$ For the case of a Raman signal of an ideal harmonic crystal, the line shape is expected to be infinitesimally narrow; however, experiments have demonstrated that the Raman linewidth of most materials exhibits a finite width indicative of the presence of decay channels, which shorten the phonons lifetimes. ${ }^{19-21}$ One fundamental lifetime-shortening mechanism in semiconductors of diamond structure has been established to occur via the anharmonic interaction. ${ }^{20}$ In this mechanism, the Raman phonons decay into other phonons that conserve momentum and energy. Moreover, in semiconductors containing impurities and defects it has been found that the these imperfections affect the Raman linewidth; thus, the contribution of the impurity shortening-lifetime mechanism has to be taken into account. ${ }^{21-22}$

In this paper, we present the study of the roomtemperature lifetimes of the Raman phonons in AlN and $\mathrm{GaN}$ materials. The Raman modes investigated in the study are the $E_{2}^{1}, E_{2}^{2}, E 1(\mathrm{TO}), A 1(\mathrm{TO})$, and $A 1(\mathrm{LO})$ modes. The $A 1(\mathrm{LO})$ mode of the GaN crystallite was not observed in the Raman spectra and its absence is attributed to the high freecarrier concentration of that crystallite; the free carriers form plasmon waves that interact and in turn damp the LO phonons. ${ }^{2,7}$ However, due to the insulating properties of the AlN, the A1(LO) mode was observed in the spectra of the crystallites. For the AlN and GaN crystallites we found that the phonons can be separated into two time regimes: a long lifetime range of the $E_{2}^{1}$ phonon, and a much shorter lifetime range, which characterizes phonons including the $E_{2}^{2}$, $E 1$ (TO), and the $A 1$ (TO). Moreover, the $A 1(\mathrm{LO})$ phonon of the AlN was found to have the shortest value among the AlN modes. In addition, we investigated two AlN crystallites that differ in their impurity concentration and found that the phonon lifetimes were correlated with the impurity concentrations. Also, we studied the $E_{2}^{2}$ phonon lifetime of highquality $\mathrm{GaN}$ film at room temperature and at $10 \mathrm{~K}$. Our results indicate that the phonon lifetime of the film is similar to that of the crystallite; moreover no significant difference 
between the lifetimes at the two temperature ranges has been observed.

The A1(LO) mode lifetime has been considered theoretically for wurtzite-based structures; ${ }^{23}$ this model predicts a three-phonon anharmonic process in which the $A 1(\mathrm{LO})$ decays into an $E 1$ (TO) phonon and a LA phonon. Our experimental measurements of this lifetime are consistent with the prediction of the model.

\section{EXPERIMENT}

Single crystals of $\mathrm{GaN}$ and AlN were grown via the sublimation method described in detail in Refs. 24-26. A short summary outlined herein highlights the findings presented in these references that are relevant to our present studies. The GaN hexagonal crystallites are colorless and secondary ion mass spectroscopy (SIMS) indicated that the concentration of all impurities, with the exception of oxygen, were at background levels; the oxygen concentration was $3 \times 10^{18}$ atoms $/ \mathrm{cm}^{3}$. Moreover, photoluminescence and transmission spectroscopies indicated that the GaN crystallites were of high-optical quality and no deep-level impurities were present.

Two different qualities of hexagonal AlN crystallites were also grown via the sublimation method: ${ }^{24}$ one type of crystallites was grown in the $2100-2250^{\circ} \mathrm{C}$ temperature range, of blue color, and will be referred to as such in this paper. The other type of crystallites, that will be referred as the transparent crystallites, were grown in the $1950-2050{ }^{\circ} \mathrm{C}$ range. SIMS analysis confirmed that the transparent crystallites contain about two orders of magnitude less $\mathrm{Si}$ and $\mathrm{C}$ impurities than the blue AlN. Oxygen impurity concentrations are similar in both samples. The $\mathrm{Si}$ and $\mathrm{C}$ impurities were incorporated into the crystals from the $\mathrm{SiC}$ seed substrates while the oxygen was incorporated from the growth environment. The transmission electron microscopy images of the AlN crystallites indicated that they are of high quality; no high-angle boundaries, stacking faults, or twinned region were observed. In addition the x-ray diffraction indicated that the residual stress level in the crystallites is low. The average size of the AlN and the GaN hexagonal crystallites is $\sim 500 \times 50 \mu \mathrm{m}$.

The micro-Raman scattering experiments were carried out at room temperature utilizing the $514.5-\mathrm{nm}$ line of the argon ion continuous laser, and the J-Y U1000 scanning double monochromator. The spot size of the laser on the samples was $\sim 2 \mu \mathrm{m}$ in diameter. The experimental error of the Raman data is $\pm 0.2 \mathrm{~cm}^{-1}$. The data were acquired in a back scattering geometry from the $a$ face of the AlN and GaN crystallites for the observation the $E_{2}^{1}, E_{2}^{2}, E 1(\mathrm{TO})$, and $A 1$ (TO) modes, and from the $c$ face for the observation of the $A 1(\mathrm{LO})$ mode. The cold temperature measurements were carried out at the macromode of spot size $\sim 1 \mathrm{~mm}$ in diameter. The error in the lifetime values, which is partially due to the experimental error and partially due to the line-shape data fit, was estimated to be $\pm 10 \%$.

\section{RESULTS AND DISCUSSION}

In order to obtain the phonon lifetime via the Raman spectral linewidth, the linewidth has to be corrected for the

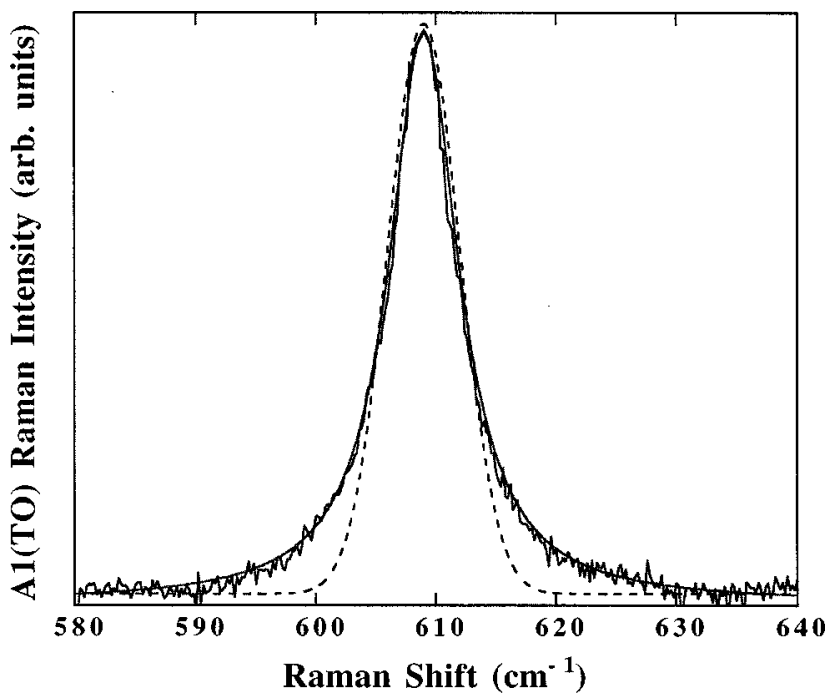

FIG. 1. The Raman spectrum (at $100-\mu \mathrm{m}$ slit width) of the A1(TO) mode of the AlN crystallite. The line and the dashed line are the Lorentzian and the Gaussian fit to the data, respectively.

contribution of the instrumental bandpass broadening. The following section describes the method used in this study to determine the actual phonon linewidth. The Raman spectra had been acquired at successive slit widths ranging from 400 $\mu \mathrm{m}$ down to $100 \mu \mathrm{m}$; the measured linewidth values $W_{M}$ were plotted as a function of the slit width $W_{S}$ and extrapolated to the zero-slit value $W_{P}$ via the relation ${ }^{27-28}$

$$
W_{M}=\sqrt{W_{P}^{2}+\left(W_{S} \times 9.2 \times 10^{-3}\right)^{2}} .
$$

The second term in Eq. (1) is the instrumental bandpass, i.e., the slit width multiplied by the monochromator linear dispersion, $9.2 \times 10^{-3} \mathrm{~cm}^{-1} / \mu \mathrm{m}$. This method yields the actual phonon linewidth $W_{P}$ from which the phonon lifetime can be calculated. However, Eq. (1) is an approximation that can be used only when the line shape of the instrument bandpass can be approximated by a Gaussian and that of the phonon by a Lorentzian. ${ }^{27-28}$ To check the bandpass characteristics of our Raman system, a spectrum of a high-quality diamond crystal (first checked to be mostly Lorentzian at $100 \mu \mathrm{m}$ slits and $W_{P} \sim 1.8 \mathrm{~cm}^{-1}$ ) was acquired at a relatively open slit width of $600 \mu \mathrm{m}$ in which case the dominance of the instrumental broadening component is expected. The line shape was found to be mostly Gaussian of linewidth $5.5 \mathrm{~cm}^{-1}$, which can be attributed to instrumental broadening. Moreover, the line shapes of the nitride crystals studied here exhibit mainly a Lorentzian component at the lower slit values as depicted in Fig. 1. These experimental results validate the appropriateness of using Eq. (1).

In general, the zero-slit Raman linewidth arises from a convolution of all broadening mechanisms, which in turn are due to the lifetime-shortening mechanisms occurring in a given crystal. ${ }^{21}$ The two main mechanisms controlling the phonon lifetimes are the phonon anharmonic interactions in which a phonon decays into other phonons, and the phonon scattering at impurity or defect centers, which is sometimes treated as a source of the inhomogeneous broadening. In the subsequent discussion, we present a study of lifetimeshortening mechanisms in two types of AlN crystallites, 


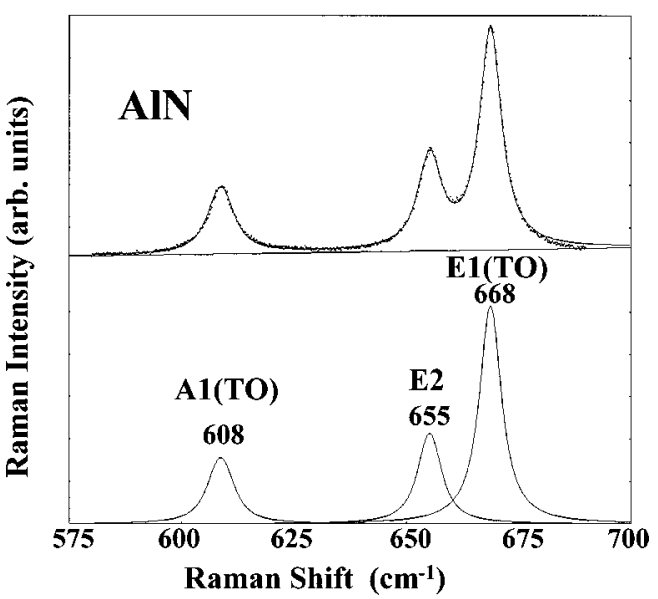

FIG. 2. The Raman spectra and the curve fit to the spectra of the transparent AlN crystallite (upper figure). The deconvoluted spectra is presented at the lower figure.

which differ mainly in their impurity contents. Figures 2 and 3 depict the Raman spectra along with the deconvoluted spectra of the AlN crystallites. The spectra presented in Fig. 2 were acquired from a transparent AlN crystallite while those in Fig. 3 are from a blue AlN crystallite. As was discussed in the experimental section, the blue crystallite contains about two orders of magnitude more $\mathrm{Si}$ and $\mathrm{C}$ impurities then the transparent AlN. In both spectra the $A 1(\mathrm{TO})$, $E_{2}^{2}$, and $E 1(\mathrm{TO})$ Raman modes are at 608,655 , and 668 $\mathrm{cm}^{-1}$ respectively. No peak shift difference between the two spectra that exceeds the experimental error of $\pm 0.2 \mathrm{~cm}^{-1}$ is evident. Thus, both samples are similar in their high quality of crystallinity and low levels of residual stress state. Moreover, the impurities have no significant effect on the residual stress. Figure 4 depicts the $E_{2}^{1}$ Raman mode at $246 \mathrm{~cm}^{-1}$ for both crystallites, and Fig. 5 presents the spectra of the $A 1$ (LO) mode at $890 \mathrm{~cm}^{-1}$.

In order to calculate the phonons lifetime via the Raman linewidths, the linewidths were plotted as a function of the slit width and a curve fit was obtained via Eq. (1). The results are presented in Fig. 6: the dots represent the data taken from the transparent AlN and the squares data from the blue

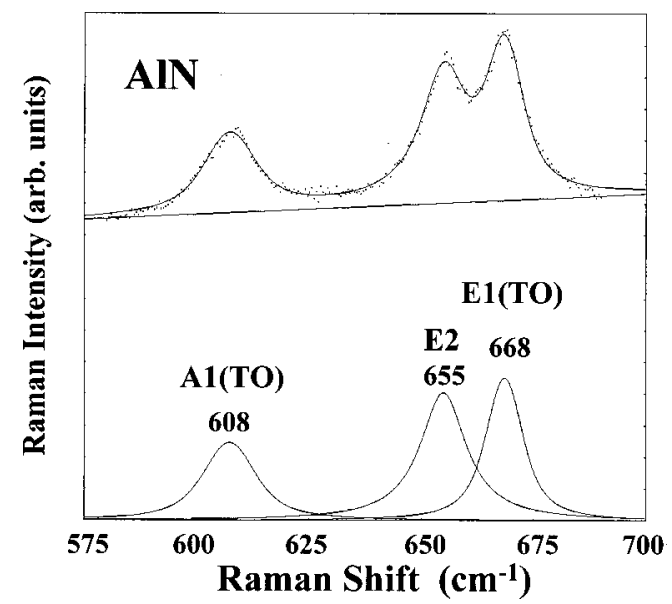

FIG. 3. The Raman spectra and the curve fit to the spectra of the blue AlN crystallite (upper figure). The deconvoluted spectra is presented at the lower figure.

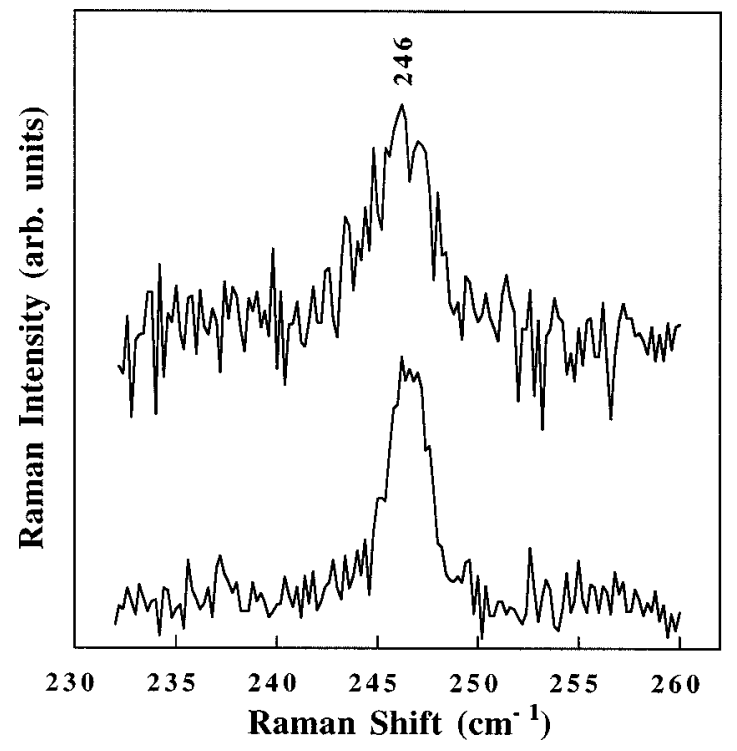

FIG. 4. The Raman spectra of the $E_{2}^{1}$ mode of the transparent AIN (lower spectrum), and of the blue crystallite.

crystallite. It is evident from the figure that the linewidth of all the Raman modes are strongly correlated with the impurity concentration. The values of the Raman linewidths at the zero slit width may be used to evaluate the phonons lifetime $\tau$ via the energy-time uncertainty relation, ${ }^{18}$

$$
\frac{\Delta E}{\hbar}=\frac{1}{\tau},
$$

where $\Delta E$ is the Raman linewidth in units of $\mathrm{cm}^{-1}$, and $\hbar$ $=5.3 \times 10^{-12} \mathrm{~cm}^{-1} \mathrm{~s}$. Table I summarizes the results: for each sample the zero slit linewidth is presented along with the calculated lifetime for each of the Raman modes. Two main conclusions can be drawn from the above results: the lifetimes are correlated with the impurity concentration, and the $E_{2}^{1}$ mode of both samples has a significantly longer lifetime than those of the $E_{2}^{2}, E 1(\mathrm{TO}), A 1(\mathrm{TO})$, and $A 1(\mathrm{LO})$ modes.

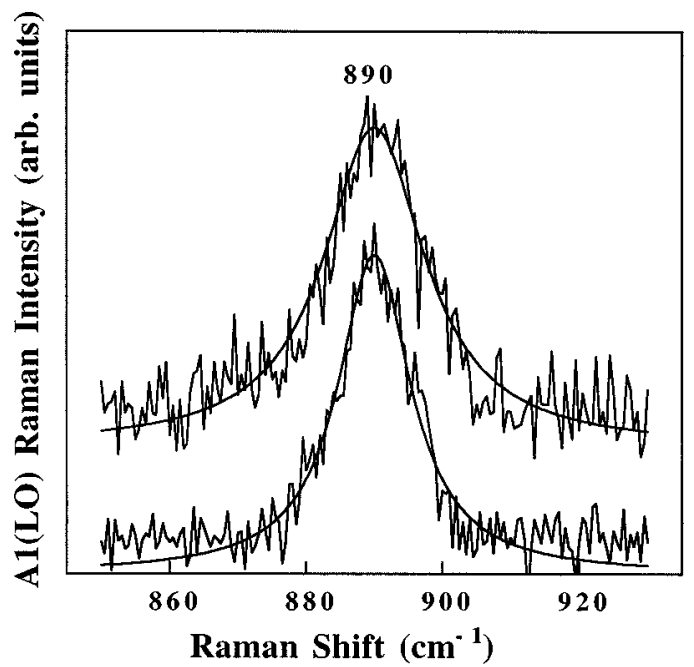

FIG. 5. The Raman spectra of the $A 1$ (LO) mode of the transparent AlN (lower spectrum), and of the blue crystallite. 


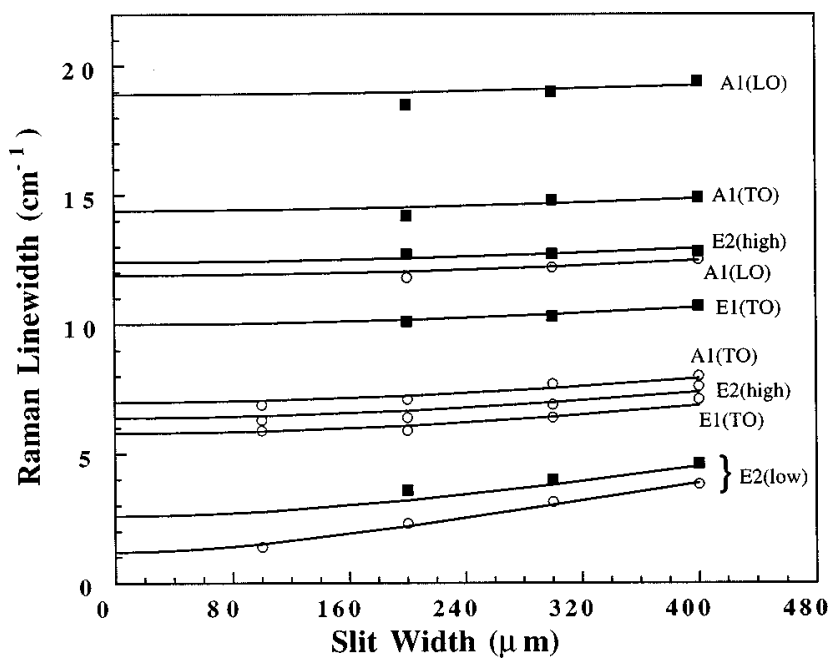

FIG. 6. The linewidth of the Raman mode as a function of the slit width. The squares represent the data of the blue AlN, and the dots of the transparent AlN. The lines are the fit to the data using Eq. (1).

The first finding may be indicative of the existence of a phonon-shortening lifetime mechanism via impurities and will be discussed next. One fundamental mechanism that affects phonon lifetimes in semiconductors is the anharmonic decay of a phonon into other Brillouin zone phonons such that the energy and momentum is conserved in the process. ${ }^{20}$ However, other additional channels of phonon decay are clearly possible especially in material containing impurities and defects. ${ }^{5,21}$ These crystal imperfections can destroy the translational symmetry of the crystal, and thus, perturb the characteristic lifetime of the propagating phonons. Our findings indicate that both channels for the phonons decay exist in the AlN crystallites; therefore, the measured lifetime $\tau$ in Eq. (1) may be given by ${ }^{18}$

$$
\frac{1}{\tau}=\frac{1}{\tau_{A}}+\frac{1}{\tau_{I}},
$$

where $\tau_{A}$ and $\tau_{1}$ are the characteristic decay times due to the crystal anharmonicity and impurity scattering, respectively. It is not a straightforward task to deconvolute the shortening mechanisms of the phonon lifetimes but the following approximated treatment gives an estimate for $\tau_{1}$. Specifically, due to its high-crystal quality and relatively low-impurity content, the measured phonon lifetimes of the transparent crystallite can be assumed to be controlled mainly by the anharmonic interaction and thus to be equal to the value of $\tau_{A}$ of each of the phonons. Using the values of $\tau_{A}$ as the characteristic anharmonic decay times of $\mathrm{AlN}$, the decay times due to impurities in the blue crystallite can be calculated via Eq. (3). The approximate method yields values of $\tau_{1}$ of $3.7,0.89,1.27,0.72$, and 0.74 ps for $E_{2}^{1}, E_{2}^{2}, E 1(\mathrm{TO})$, $A 1$ (TO), and $A 1$ (LO) modes, respectively.

The anharmonic decay of phonons has been studied extensively for zinc-blend semiconductors ${ }^{20,29-30}$ but has received considerably less attention for wurtzite structure materials such as $\mathrm{Al}_{x} \mathrm{Ga}_{1-x} \mathrm{~N} .{ }^{23,31}$ Of special significance for the nitride-based wurtzite structures is the decay of the LOphonon modes, which dominates in the polar-optical-phonon carrier interaction in many high-speed optoelectronic devices. ${ }^{32-33}$ The accepted model, known as Klemens' channel, of the LO-phonon decay in the cubic structure materials has been established to occur via the annihilation of the LO Raman phonon (of frequency $\omega_{\mathrm{LO}}$ ) and the creation of two LA phonons (each of frequency $\omega_{\mathrm{LA}}$ ) at the zone edge. ${ }^{20}$ In this model, a special case of the three-phonon process, energy conservation requires that $2 \omega_{\mathrm{LA}}=\omega_{\mathrm{LO}}$. However, in $\mathrm{AlN}$, due to the substantial mass difference between the $\mathrm{Al}$ and the $\mathrm{N}$ elements a large gap in the AlN phonon spectrum exists: the $A 1$ (LO) frequency at the $\Gamma$ point is at $\sim 890 \mathrm{~cm}^{-1}$ while the LA at zone edge has frequency $\sim 400 \mathrm{~cm}^{-1}$. 34 Accordingly, the Klemens' channel is not applicable here since $2 \omega_{\mathrm{LA}}<\omega_{\mathrm{LO}}$. A four-phonon interaction would permit the conservation of energy; however, this interaction would result in an unreasonably long lifetime on the order of two or three orders of magnitude longer than that predicted for the three-phonon process. ${ }^{23}$ To account for the lifetime of the LO in the wurtzite materials, a three-phonon model has been proposed recently in which the LO mode decays into a TO mode and an LA mode; this decay route results in a more reasonable lifetime of a few picoseconds. ${ }^{23}$ This model has been supported by time-resolved Raman measurements of the $A 1$ (LO) phonon lifetime in wurtzite GaN thin films. ${ }^{31}$ Moreover, our experimental results of the $A 1(\mathrm{LO})$ lifetimes of AlN crystallites $(\sim 0.5 \mathrm{ps})$ are in accord with the time scale of the three-phonon anharmonic process, if we include impurity scattering as an additional shortening mechanism.

In order to further investigate the relatively long lifetime of the $E_{2}^{1}$ mode, the Raman modes of $\mathrm{GaN}$ as well as $\mathrm{ZnO}$ crystals were analyzed. Figures 7 and 8 depict the Raman

TABLE I. The zero-slit width Raman linewidths and the corresponding phonon lifetimes of the AlN, $\mathrm{GaN}$, and $\mathrm{ZnO}$ crystallites.

\begin{tabular}{|c|c|c|c|c|c|c|c|c|}
\hline \multirow[b]{2}{*}{$\begin{array}{l}\text { Raman } \\
\text { modes }\end{array}$} & \multicolumn{2}{|c|}{$\begin{array}{c}\text { AlN } \\
\text { transparent }\end{array}$} & \multicolumn{2}{|c|}{$\begin{array}{l}\text { AlN } \\
\text { blue }\end{array}$} & \multicolumn{2}{|c|}{$\mathrm{GaN}$} & \multicolumn{2}{|c|}{$\mathrm{ZnO}$} \\
\hline & $\begin{array}{c}\Gamma \\
\left(\mathrm{cm}^{-1}\right)\end{array}$ & $\begin{array}{c}\tau \\
10^{-12} \mathrm{~s}\end{array}$ & $\begin{array}{c}\Gamma \\
\left(\mathrm{cm}^{-1}\right)\end{array}$ & $\begin{array}{c}\tau \\
10^{-12} \mathrm{~s}\end{array}$ & $\begin{array}{c}\Gamma \\
\left(\mathrm{cm}^{-1}\right)\end{array}$ & $\begin{array}{c}\tau \\
10^{-12} \mathrm{~s}\end{array}$ & $\begin{array}{c}\Gamma \\
\left(\mathrm{cm}^{-1}\right)\end{array}$ & $\begin{array}{c}\tau \\
10^{-12}\end{array}$ \\
\hline$E_{2}^{1}$ & 1.2 & 4.4 & 2.6 & 2.0 & 0.5 & 10.1 & 0.9 & 5.9 \\
\hline$E_{2}^{2}$ & 6.4 & 0.83 & 12.4 & 0.43 & 3.9 & 1.4 & 5.9 & 0.9 \\
\hline$E 1(\mathrm{TO})$ & 5.8 & 0.91 & 10.0 & 0.53 & 5.6 & 0.95 & & \\
\hline$A 1(\mathrm{TO})$ & 7.0 & 0.76 & 14.4 & 0.37 & 11.6 & 0.46 & & \\
\hline$A 1(\mathrm{LO})$ & 11.9 & 0.45 & 18.9 & 0.28 & & & & \\
\hline
\end{tabular}




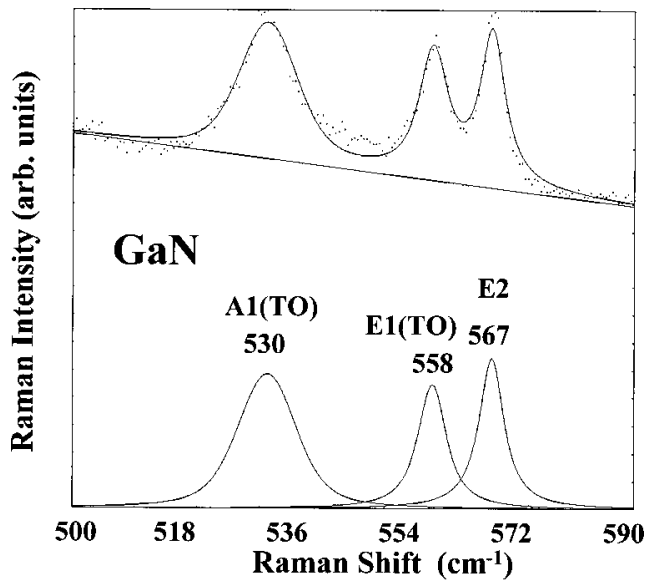

FIG. 7. The Raman spectra, the curve fit, and the deconvolution spectra of the GaN crystallite.

spectra of the GaN crystallite: the $A 1(\mathrm{TO}), E 1(\mathrm{TO}), E_{2}^{2}$, and $E_{2}^{1}$ modes are at 530, 558, 567, and $142 \mathrm{~cm}^{-1}$ respectively. The corresponding lifetimes computed via the previously described method are summarized in Table I. Similar to the AlN lifetimes, the $E_{2}^{1}$ mode of the GaN has a significantly longer lifetime than that of the $E_{2}^{2}, E 1(\mathrm{TO})$, and $A 1$ (TO) modes. Figure 9 depicts the linewidth behavior of the $E_{2}^{1}$ and the $E_{2}^{2}$ modes of the $\mathrm{ZnO}, \mathrm{GaN}$, and the transparent AlN crystals; the zero-slit lifetimes of the $\mathrm{ZnO}$ modes are presented in Table I. Our analyses indicate that the $E_{2}^{1}$ mode of the $\mathrm{ZnO}$ crystallite exhibits relatively long lifetime as well. As was noted previously, only the LO-mode lifetime in wurtzite structure has been considered theoretically. ${ }^{23} \mathrm{How}-$ ever, the long lifetime of the $E_{2}^{1}$ in that structure is not unexpected in view of the factors determining the anharmonic lifetimes: energy-conservation constraints, the density of the final states, and the anharmonic interaction coefficient. Unlike the other modes, the energy of the $E_{2}^{1}$ mode lies at the low-energy regime of the wurtzite dispersion curve ${ }^{34-35}$ and only the acoustical phonons are available as a channel of decay. At the zone edges the energies of the acoustical phonons are larger than that of the $E_{2}^{1}$ mode; thus, in order for the energy conservation to hold the optical phonons have to decay into acoustical phonons at the zone center for which

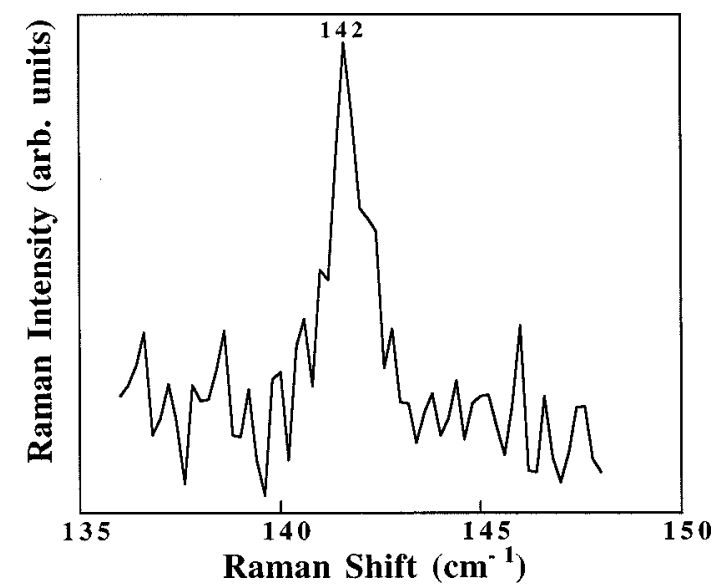

FIG. 8. The Raman spectra of the $E_{2}^{1}$ mode of the GaN crystallite.

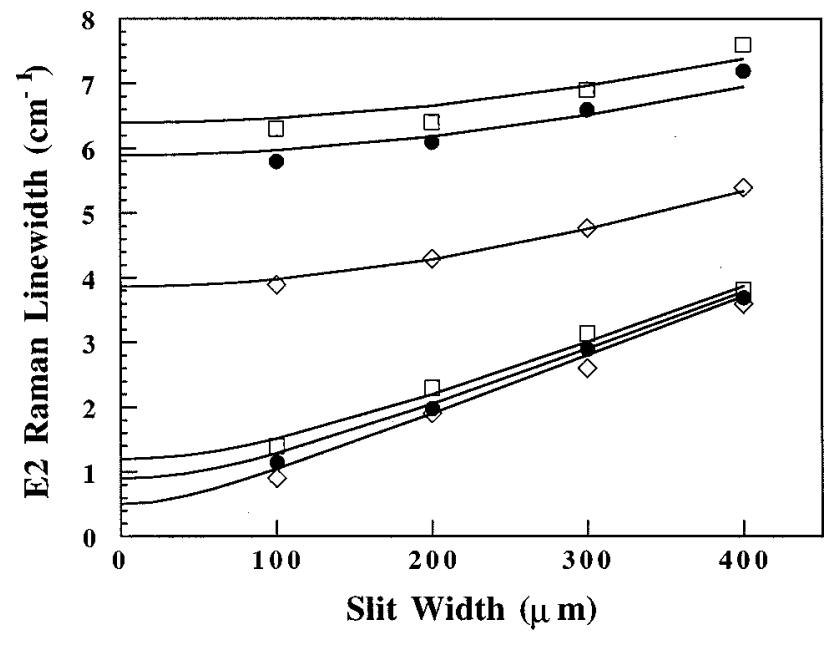

FIG. 9. The $E_{2}^{1}$ modes (lower curves) and the $E_{2}^{2}$ modes (upper curves) of the blue AlN (squares), $\mathrm{ZnO}$ (circles), and GaN (diamonds) as a function of the slit width.

their density is low. Although the contribution of the anharmonic coefficient, which to our knowledge has not been yet determined theoretically, has to be taken into account, we speculate that the low density of state significantly reduces the scattering rate, thus increasing the phonon lifetime.

Lastly, we investigated the $E_{2}^{2}$ mode of GaN film. Figure 10 depicts the Raman spectra of a 3- $\mu \mathrm{m}$ undoped GaN film grown on $\mathrm{SiC}$ substrate with an AlN buffer layer and of a free-carrier concentration $\sim 10^{16} \mathrm{~cm}^{-3}$. $^{7}$ The spectra were acquired in a backscattering geometry at room temperature (RT) and at $10 \mathrm{~K}$; the slit width was set at $200 \mu \mathrm{m}$. The line shape of both lines is a Lorentzian with a linewidth of 4 and $3.2 \mathrm{~cm}^{-1}$ for the RT and the $10 \mathrm{~K}$ spectrum, respectively. Using Eqs. (1) and (2) the zero-slit values and the lifetimes may be calculated. The RT and the $10 \mathrm{~K}$ lifetimes were found to be 2 and $1.5 \mathrm{ps}$, respectively. Our results indicate that the lifetime of the $E_{2}^{2}$ mode of the GaN film is similar to that of the crystallite $(1.4 \mathrm{ps})$. Furthermore, no significant

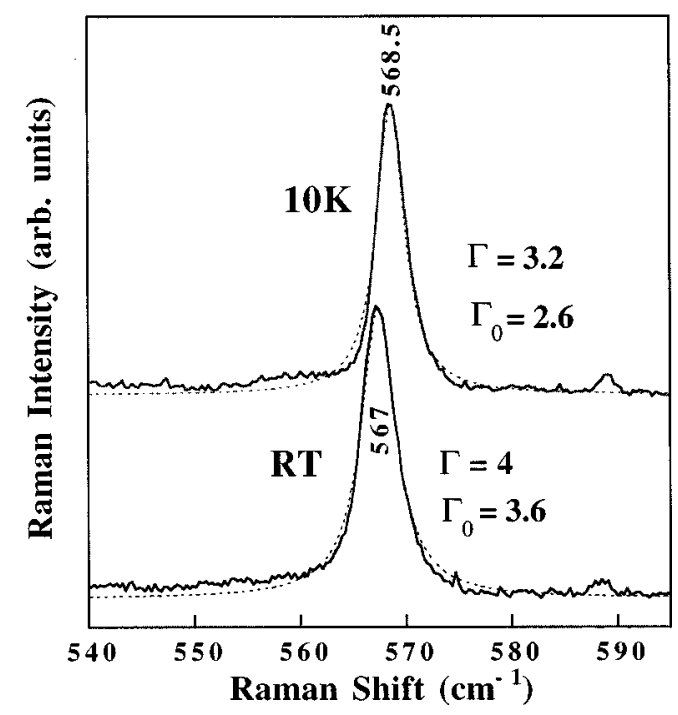

FIG. 10. The spectra (200- $\mu \mathrm{m}$ slit width) of $E_{2}^{2}$ modes of GaN film at room temperature and at $10 \mathrm{~K}$. The dashed line represents the Lorentzian fit to the data. 
lifetime change occurs at $10 \mathrm{~K}$. A weak and broad $A 1(\mathrm{LO})$ Raman line from the film was observed in the spectra; however, due to the strong SiC Raman signal that appears in the same energy range, an accurate analysis of the $A 1(\mathrm{LO})$ lifetime was not possible. The lifetime of the $A 1(\mathrm{LO})$ was obtained previously via time-resolved Raman measurements; the reported lifetime was $\sim 3$ ps at room temperature. ${ }^{31}$ This lifetime value is somewhat large compared to our values of mode lifetimes in the short-lifetime regime of the GaN crystallite (see Table I).

\section{CONCLUSIONS}

The lifetimes of the Raman phonons of $\mathrm{GaN}$ and AlN were found to exhibit two time regimes: the long lifetime range of the $E_{2}^{1}$ phonons and the much shorter lifetime range, which includes the $E_{2}^{2}, E 1(\mathrm{TO})$, and $A 1(\mathrm{TO})$ phonons. The lifetime of the $A 1(\mathrm{LO})$ mode of AlN crystallites were found to be the shortest among the modes studied here. The $E_{2}^{1}$ lifetime of high-quality and of relatively low-impurity concentration AlN crystallite is $4.4 \mathrm{ps}$, which is about five times longer than that of the $E_{2}^{2}, E 1(\mathrm{TO})$, and $A 1(\mathrm{TO})$ modes and about an order of magnitude longer than that of the $A 1$ (LO) mode. The lifetime of the $E_{2}^{1}$ of $\mathrm{GaN}$ crystallite is $10.1 \mathrm{ps}$, which is about ten times longer than the other modes. We also investigated two AlN crystallites, which differ in their impurity concentration and found that the phonon lifetimes were correlated with the impurity concentrations. The effect of the two orders of magnitude increase in relative impurity concentration shortened the lifetime of the phonons by $\sim 50 \%$. Additionally, we studied the $E_{2}^{2}$ phonon lifetime of high-quality $\mathrm{GaN}$ film at room temperature and at $10 \mathrm{~K}$. Our results indicate that the phonon lifetime of the film is similar to that of the crystallite; moreover, no significant difference between the lifetimes at the two temperature ranges was observed.

\section{ACKNOWLEDGMENTS}

We gratefully acknowledge the Army Research Office for supporting this study. L. Bergman acknowledges, additionally, the National Research Council for supporting this research via the Research Associateship Award program. We have also benefited from the ongoing collaboration with Professor Ki Wook Kim and his group in the Department of Electrical and Computer Engineering at the North Carolina State University.
${ }^{1}$ Properties of Group III Nitrides, edited by J. H. Edgar (INSPEC, London, 1994).

${ }^{2}$ T. Kozawa, T. Kachi, H. Kano, Y. Taga, M. Hashimoto, N. Koide, and K. Manabe, J. Appl. Phys. 75, 1098 (1994).

${ }^{3}$ L. Bergman and R. J. Nemanich, Annu. Rev. Mater. Sci. 26, 551 (1996).

${ }^{4}$ C. A. Arguello, D. L. Rousseau, and S. P. S. Porto, Phys. Rev. 181, 1351 (1969).

${ }^{5}$ J. M. Zhang, T. Ruf, M. Cardona, O. Ambacher, M. Stutzmann, J. M. Wagner, and F. Bechstedt, Phys. Rev. B 56, 14399 (1997).

${ }^{6}$ L. Bergman, M. D. Bremser, W. G. Perry, R. F. Davis, M. Dutta, and R. J. Nemanich, Appl. Phys. Lett. 71, 2157 (1997).

${ }^{7}$ L. Bergman, M. D. Bremser, J. A. Christman, S. W. King, R. F. Davis, and R. J. Nemanich, in III-V Nitrides, edited by F. A. Ponce et al., MRS Symposia Proceedings No. 449 Symp. (Materials Research Society, Pittsburgh, 1997), p. 725.

${ }^{8}$ F. Demangeot, J. Groenen, J. Frandon, M. A. Renucci, O. Briot, S. Clur, and R. L. Aulombard, Appl. Phys. Lett. 72, 2674 (1998).

${ }^{9}$ S. Murugkar, R. Merlin, A. Botchkarev, A. Salvador, and H. Morkoc, J. Appl. Phys. 77, 6042 (1995).

${ }^{10}$ C. Wetzel, E. E. Haller, H. Amano, and I. Akasaki, Appl. Phys. Lett. 68, 2547 (1996).

${ }^{11}$ M. A. Stroscio, J. Appl. Phys. 80, 6864 (1996); H. B. Teng, J. P. Sun, G. I. Haddad, M. A. Stroscio, SeGi Yu, and K. W. Kim, ibid. 84, 2155 (1998).

${ }^{12}$ M. V. Kisin, V. B. Gorfinkel, M. A. Stroscio, G. Belenky, and S. Luryi, J. Appl. Phys. 82, 2031 (1997).

${ }^{13}$ L. Bergman and R. J. Nemanich, J. Appl. Phys. 78, 6709 (1995).

${ }^{14}$ E. S. Zouboulis and M. Grimsditch, Phys. Rev. B 43, 12490 (1991).

${ }^{15}$ H. Herchen and M. A. Cappelli, Phys. Rev. B 47, 14193 (1993).

${ }^{16}$ T. R. Hart, R. L. Aggarwal, and B. Lax, Phys. Rev. B 1, 638 (1970).
${ }^{17}$ E. T. M. Kernohan, R. T. Phillips, B. H. Bairamov, D. A. Ritchie, and M. Y. Simmons, Solid State Commun. 100, 263 (1996).

${ }^{18}$ B. Di Bartolo, Optical Interactions in Solids (Wiley, New York, 1968).

${ }^{19}$ J. Menendez and M. Cardona, Phys. Rev. B 29, 2051 (1984).

${ }^{20}$ P. G. Klemens, Phys. Rev. 148, 845 (1966).

${ }^{21}$ A. A. Maradudin, in Solid State Physics, Advances in Research and Applications, edited by E. Seitz, and D. Turnbull (Academic, New York, 1966), Vol. 18, p. 273.

${ }^{22}$ L. E. McNeil, M. Grimsditch, and R. H. French, J. Am. Ceram. Soc. 76, 1132 (1993).

${ }^{23}$ B. K. Ridley, J. Phys.: Condens. Matter 8, L511 (1996).

${ }^{24}$ C. M. Balkas, Z. Sitar, T. Zheleva, L. Bergman, R. J. Nemanich, and R. F. Davis, J. Cryst. Growth 179, 363 (1997).

${ }^{25}$ C. M. Balkas, Z. Sitar, L. Bergman, I. K. Shmagin, J. F. Muth, R. Kolbas, R. J. Nemanich, and R. F. Davis, J. Cryst. Growth (to be published).

${ }^{26}$ C. M. Balkas, Z. Sitar, T. Zheleva, L. Bergman, I. K. Shmagin, J. F. Muth, R. Kolbas, R. J. Nemanich, and R. F. Davis, in $I I I-V$ Nitrides (Ref. 7), p. 41.

${ }^{27}$ H. Bernstein and G. Allen, J. Opt. Soc. Am. 45, 237 (1955).

${ }^{28}$ E. E. Whiting, J. Quant. Spectrosc. Radiat. Transf. 8, 1379 (1968).

${ }^{29}$ A. Debernardi, Phys. Rev. B 57, 12847 (1998).

${ }^{30}$ D. J. Ecsedy and P. G. Klemens, Phys. Rev. B 15, 5957 (1977).

${ }^{31}$ K. T. Tsen, D. K. Ferry, A. Botchkarev, B. Sverdlov, A. Salvador, and H. Morkoc, Appl. Phys. Lett. 72, 2132 (1998).

${ }^{32}$ B. C. Lee, K. W. Kim, M. Dutta, and M. A. Stroscio, Phys. Rev. B 56, 997 (1997).

${ }^{33}$ B. C. Lee, K. W. Kim, M. A. Stroscio, and M. Dutta, Phys. Rev. B 58, 4860 (1998).

${ }^{34}$ J. C. Nipko and C. K. Loong, Phys. Rev. B 57, 10550 (1998).

${ }^{35}$ A. W. Hewat, Solid State Commun. 8, 187 (1970). 